\title{
Another proof of the Harer-Zagier formula
}

\author{
Boris Pittel \\ Department of Mathematics, Ohio State University, \\ 231 West 18th Avenue, Columbus, OH 43210, USA
}

Submitted: Jul 17, 2015; Accepted: Jan 21, 2016; Published: Feb 5, 2016

Mathematics Subject Classifications: 05A15, 05A16, 05A19, 05D40, 05E10, 20P05, 60B15, $60 \mathrm{C} 05$

\begin{abstract}
For a regular $2 n$-gon there are $(2 n-1) ! !$ ways to match and glue the $2 n$ sides. The Harer-Zagier bivariate generating function enumerates the gluings by $n$ and the genus $g$ of the attendant surface and leads to a recurrence equation for the counts of gluings with parameters $n$ and $g$. This formula was originally obtained using multidimensional Gaussian integrals. Soon after, Jackson and later Zagier found alternative proofs using symmetric group characters. In this note we give a different, characters-based, proof. Its core is computing and marginally inverting the Fourier transform of the underlying probability measure on $S_{2 n}$. A key ingredient is the Murnaghan-Nakayama rule for the characters associated with one-hook Young diagrams.
\end{abstract}

Keywords: Keywords: surfaces, chord diagrams, genus, random permutations, Fourier transform, irreducible characters, Murnaghan-Nakayama, generating functions

\section{Introduction and main results}

Consider a regular, oriented, $2 n$-gon. There are $(2 n-1)$ !! ways to match and glue $2 n$-sides observing head-to-tail constraint in each glued pair. Each such gluing produces an oneface map on an oriented surface. Let $\varepsilon_{g}(n)$ denote the total number of gluings resulting in a surface of genus $g$. Thirty years ago Harer and Zagier [9] discovered that

$$
1+2 x y+2 \sum_{n=1}^{\infty} \frac{x^{n+1}}{(2 n-1) ! !} \sum_{g} \varepsilon_{g}(n) y^{n+1-2 g}=\left(\frac{1+x}{1-x}\right)^{y}
$$

here $n+1-2 g$ is the number of vertices in the map on the surface. Of course, $\varepsilon_{g}(n) /(2 n-$ $1)$ !! is the probability that the uniformly random gluing generates a surface of genus $g$. So, introducing the random variable $G_{n}, n \geqslant 1$, genus of the random surface, so that 
$V_{n}:=n+1-G_{n}$ is the number of vertices on the surface map, and setting $G_{0}=0$, we rewrite (1.1) as

$$
1+2 \sum_{n=0}^{\infty} x^{n+1} \mathrm{E}\left[y^{n+1-2 G_{n}}\right]=\left(\frac{1+x}{1-x}\right)^{y} .
$$

Their proof used a powerful technique based on multidimensional integrals with respect to a Gaussian measure on $\mathbb{R}^{k}$. The identity (1.1) implied a remarkable 3-term recurrence for the counts $\varepsilon_{g}(n)$. A year later Jackson [11] found a group characters-based derivation of an explicit formula for those counts, expressed through Stirling numbers of both kinds, and used it to prove the recurrence anew. Subsequently Itzykson and Zuber [10] reduced the combinatorial calculations in [9]. In 1995 Zagier found another, shorter, proof of (1.1) based on group characters, see [18] and also Zagier's Appendix to the book [12] by Lando and Zvonkin. In 2001 Lass [13] gave a combinatorial derivation based upon the enumeration of arborescences and Euler circuits.

The Harer-Zagier formula was used by Linial and Nowik [14] to obtain a sharp asymptotic formula for $\mathrm{E}\left[G_{n}\right]$ and later by Chmutov and Pittel [1] to prove that $G_{n}$ is asymptotically Gaussian with mean $(n-\log n) / 2$ and variance $(\log n) / 4$. Pippenger and Schleich [15], Gamburd [7], Fleming and Pippenger [6] studied the random surface obtained by gluing together edge-wise $n$ oriented polygonal disks, all with the same number of sides, 3 in [15], and $k \geqslant 3$ in [7], [6]; $k n$ needs to be even, of course. In addition to the uniformity of the "gluing" permutation $\alpha$, Gamburd also assumed that those oriented $k$-gons were the cycles of the permutation $\beta$ chosen independently of $\alpha$ and uniformly at random among all permutations with $k$-long cycles only, rather than the cycles of a fixed permutation $\beta$. Fleming and Pippenger showed that the counts of cycles by size in the two resulting permutations $\gamma:=\alpha \beta$ are equidistributed. Gamburd used a Fourier transform-based bound for the total variation distance between two probability measures on a finite group, due to Diaconis and Shahshahani [4], [5], to prove that, when $2 \operatorname{lcm}\{2, k\} \mid k n, \gamma$ is asymptotically uniform on the alternating subgroup $A_{k n}$. Fleming and Pippenger used Gamburd's result to obtain very sharp approximations for the moments of $V_{n}$, confirming the conjectures made in [15] for the case $k=3$. Thus the number of vertices $V_{n}$ in the surface obtained by gluing the given discs and the number of cycles of $\gamma$ are equidistributed. Chmutov and Pittel [2] extended Gamburd's result to the general case of $n$ polygons with arbitrary "circumferences", adding up to an even $N \rightarrow \infty: \gamma$ is asymptotically uniform on $A_{N}$ (on $A_{N}^{c}$ resp.) if $N-n$ and $N / 2$ are of the same (opposite resp.) parity.

In this note we combine Gamburd's ideas and the Fourier transform on $S_{N}$ to give a new proof of the identity (1.2). Aside from Murnaghan-Nakayama rule, mostly for a simple case of the one-hook diagrams, our relatively short argument is based on elementary enumeration. We hope to apply analogous techniques to other models of random surfaces.

\section{Derivation of Harer-Zagier formula}

For an even $N=2 n$, consider a directed polygon with $N$ sides labeled by elements of $[N]$, and let $\alpha$ be a fixed-point free involution of $[N]$, i.e. a permutation of $N]$ with all cycles 
of length 2. We can view the polygon as a unicyclic permutation $\beta$ of $[N]$. Here is how the pair $(\alpha, \beta)$ induces the corresponding surface. The first edge $e_{1}$ is glued, head-to-tail, to the edge $\beta\left(e_{1}\right)$; the edge $\beta\left(e_{1}\right)$ is followed by the edge $e_{2}=\alpha\left(\beta\left(e_{1}\right)\right)=(\alpha \beta)\left(e_{1}\right)$ in the cycle $\beta$. Next $e_{2}$ is glued to $\beta\left(e_{2}\right)$ followed by $e_{3}=\alpha\left(\beta\left(e_{2}\right)\right)=(\alpha \beta)\left(e_{2}\right)$ in the the cycle $\beta$. This process produces a sequence of edges $e_{1}, e_{2}, \ldots$, whose tails collapse into a single vertex of the map. Since $\alpha \beta$ is a permutation of $[N]$, the sequence of edges eventually loops back on the starting edge $e_{1}$, forming a cycle $e_{1} \rightarrow e_{2} \rightarrow \cdots \rightarrow e_{m} \rightarrow e_{1}$ of $\alpha \beta$. Next, starting from the first edge not in this cycle, i.e. distinct from $e_{1}, \ldots, e_{m}$, we obtain a cycle with a disjoint support, containing this edge, that determines another vertex of the map. Proceeding in the same way, we will eventually partition the edge set $[N]$ into disjoint subsets, each associated with its own vertex of the map. Clearly, the number of those subsets, i.e. the number of vertices $V$, equals the number of cycles of $\gamma=\alpha \beta$. Since the number of edges of the map is $N / 2=n$, and the number of faces is 1 , the Euler characteristic $\chi=V-N+1$, and the genus $g=1-\frac{\chi}{2}$, so that $V=n+1-2 g$. Thus enumerating surfaces by the genus $g$ is reduced to enumerating pairs $(\alpha, \beta)$ by the number of cycles of $\alpha \beta$.

Casting the problem in the probabilistic light, let $\alpha$ and $\beta$ be two independent random permutations of $[N]$ chosen uniformly among the permutations with all cycles of length 2 , and among all $(N-1)$ ! unicyclic permutations respectively. Our goal is to determine the generating function of $V_{n}$, the number of cycles of the random permutation $\gamma:=\alpha \beta$, in a form that allows to evaluate, rather directly, the bivariate generating function of the number of cycles, i.e. to prove the Harer-Zagier formula anew.

The starting point is the Fourier inversion formula for a general probability measure $P$ on $S_{N}$, Diaconis [5]:

$$
P(s)=\frac{1}{N !} \sum_{\lambda \vdash N} f^{\lambda} \operatorname{tr}\left(\rho^{\lambda}\left(s^{-1}\right) \hat{P}\left(\rho^{\lambda}\right)\right) ; \quad s \in S_{N} .
$$

Here $\lambda$ is a generic partition of $N, \rho^{\lambda}$ is the irreducible representation of $S_{N}$ associated with $\lambda, f^{\lambda}=\operatorname{dim}\left(\rho^{\lambda}\right)$, and $\hat{P}\left(\rho^{\lambda}\right)$ is the matrix-valued Fourier transform of $P(\cdot)$ evaluated at $\rho^{\lambda}$,

$$
\hat{P}\left(\rho^{\lambda}\right)=\sum_{s \in S_{N}} \rho^{\lambda}(s) P(s),
$$

and $\operatorname{tr}(A)$ is the trace of a square matrix $A$.

Let us evaluate the RHS of (2.1) for $P=P_{\sigma}$, the probability measure on $S_{N}$ induced by $\sigma=\prod_{j=1}^{k} \sigma_{j}$, where $\sigma_{j}$ is uniform on a conjugacy class $C_{j}$, and $\sigma_{1}, \ldots, \sigma_{k}$ are independent of each other. By independence of $\sigma_{j}$,

$$
P_{\sigma}(s)=\sum_{s_{1}, \ldots, s_{k}} \prod_{j} P_{\sigma_{j}}\left(s_{j}\right), \quad\left(s_{1} \cdots s_{k}=s\right),
$$

i.e. $P_{\sigma}$ is the convolution of $P_{\sigma_{1}}, \ldots, P_{\sigma_{k}}$. So, by multiplicativity of the Fourier transform for convolutions,

$$
\hat{P}_{\sigma}\left(\rho^{\lambda}\right)=\prod_{j} \hat{P}_{\sigma_{j}}\left(\rho^{\lambda}\right)
$$


Since each $P_{\sigma_{j}}$ is supported by the single conjugacy class $C_{j}$, we have

$$
\hat{P}_{\sigma_{j}}\left(\rho^{\lambda}\right)=\frac{\chi^{\lambda}\left(C_{j}\right)}{f^{\lambda}} I_{f^{\lambda}}
$$

$I_{m}$ being an $m \times m$ identity matrix, see [5]. So

$$
\hat{P}_{\sigma}\left(\rho^{\lambda}\right)=\prod_{j=1}^{k} \hat{P}_{\sigma_{j}}\left(\rho^{\lambda}\right)=\left(f^{\lambda}\right)^{-k} \prod_{j=1}^{k} \chi^{\lambda}\left(C_{j}\right) I_{f^{\lambda}},
$$

and (2.1) becomes

$$
\begin{aligned}
P_{\sigma}(s) & =\frac{1}{N !} \sum_{\lambda}\left(f^{\lambda}\right)^{-k+1}\left(\prod_{j=1}^{k} \chi^{\lambda}\left(C_{j}\right)\right) \operatorname{tr}\left(\rho^{\lambda}\left(s^{-1}\right) I_{f^{\lambda}}\right) \\
& =\frac{1}{N !} \sum_{\lambda}\left(f^{\lambda}\right)^{-k+1} \chi^{\lambda}(s) \prod_{j=1}^{k} \chi^{\lambda}\left(C_{j}\right) .
\end{aligned}
$$

For a special case $s=$ id $(2.2)$ becomes

$$
\mathrm{P}_{\sigma}(\mathrm{id})=\frac{1}{N !} \sum_{\lambda}\left(f^{\lambda}\right)^{-k+2} \prod_{j=1}^{k} \chi^{\lambda}\left(C_{j}\right),
$$

and since the LHS is the ratio of $\mathcal{N}\left(C_{1}, \ldots, C_{k}\right)$, the number of ways to write the identity permutation as the product of elements of $C_{1}, \ldots, C_{k}$, to $\prod_{j=1}^{k}\left|C_{j}\right|$, we obtain the $S_{N^{-}}$ version of Frobenius's identity

$$
\mathcal{N}\left(C_{1}, \ldots, C_{k}\right)=\frac{\prod_{j=1}^{k}\left|C_{j}\right|}{N !} \sum_{\lambda}\left(f^{\lambda}\right)^{-k+2} \prod_{j=1}^{k} \chi^{\lambda}\left(C_{j}\right) .
$$

Zagier's proof in [18] used (2.3) for $k=3$. In our argument we use (2.2) for $k=2$, heavily relying on arbitrariness of $s \in S_{N}$. For $\sigma_{1}=\alpha, \sigma_{2}=\beta$ and $\sigma=\gamma=\alpha \beta$, this equation becomes

$$
P_{\gamma}(s)=\frac{1}{N !} \sum_{\lambda}\left(f^{\lambda}\right)^{-1} \chi^{\lambda}(s) \chi^{\lambda}\left(\mathcal{C}_{2}\right) \chi^{\lambda}\left(\mathcal{C}_{N}\right)
$$

$\mathcal{C}_{2}\left(\mathcal{C}_{N}\right.$ resp.) consists of all $(N-1)$ !! permutations with cycles of length 2 only (all $(N-1)$ ! unicyclic permutations resp.).

Let $\mathcal{C}_{\vec{\nu}}$ be a generic conjugacy class comprising all permutations with given counts $\nu_{j}$ of cycles of length $j \in[1, N]$. Define $J=J(\vec{\nu})=\left\{j \in[1, N]: \nu_{j}>0\right\}$. Let $\boldsymbol{\alpha}=\left(\alpha_{1}, \alpha_{2}, \ldots\right)$ be an arbitrary composition of $N$ containing $\nu_{j}$ components equal $j$, $(j \in J)$. By Murnaghan-Nakayama rule, Stanley [16] (Section 7.17, Equation (7.75)),

$$
\chi^{\lambda}\left(\mathcal{C}_{\vec{\nu}}\right)=\sum_{T}(-1)^{\mathrm{ht}(T)}
$$


where the sum is over all rim hook diagrams $T$ of shape $\lambda$ and type $\boldsymbol{\alpha}$, i. e. over all ways to empty the diagram $\lambda$ by successive deletions of the rim hooks, one hook at a time, of lengths $\alpha_{1}, \alpha_{2}, \ldots$ Further ht $(T)$ is the sum of heights of the individual hooks (number of rows minus 1 ) in the hook diagram $T$.

This remarkable formula implies that $\chi^{\lambda}\left(\mathcal{C}_{N}\right)=0$ unless the diagram $\lambda$ is a single hook $\lambda^{*}$, with one row of length $\lambda_{1}$ and one column of height $\lambda^{1}, \lambda_{1}+\lambda^{1}=N+1$, in which case

$$
\chi^{\lambda}\left(\mathcal{C}_{N}\right)=(-1)^{\lambda^{1}-1}
$$

Consider $\chi^{\lambda^{*}}\left(\mathcal{C}_{2}\right)$. If $\lambda_{1}$ is even, then $\lambda^{1}$ is odd, so that for each $T$ the rim hook deleted last consists of two first cells in the row. So ht $(T)=\left(\lambda^{1}-1\right) / 2$ for every $T$, and the number of $T$ 's is the number of ways to intersperse first $\left(\lambda_{1}-2\right) / 2$ deletions, from left to right, of domino tiles in the row with $\left(\lambda^{1}-1\right) / 2$ deletions of domino tiles, from bottom to top, in the column, and this number is

$$
\left(\begin{array}{c}
\frac{\lambda_{1}-2+\lambda^{1}-1}{2} \\
\frac{\lambda_{1}-2}{2}
\end{array}\right)=\left(\begin{array}{c}
\frac{N-2}{2} \\
\frac{\lambda_{1}-2}{2}
\end{array}\right) \Longrightarrow \chi^{\lambda^{*}}\left(\mathcal{C}_{2}\right)=(-1)^{\left(\lambda^{1}-1\right) / 2}\left(\begin{array}{c}
\frac{N-2}{2} \\
\frac{\lambda_{1}-2}{2}
\end{array}\right)
$$

Analogously, if $\lambda_{1}$ is odd then

$$
\chi^{\lambda^{*}}\left(\mathcal{C}_{2}\right)=(-1)^{\lambda^{1} / 2}\left(\begin{array}{c}
\frac{N-2}{2} \\
\frac{\lambda_{1}-1}{2}
\end{array}\right) .
$$

Combining (2.6)-(2.8), we have

$$
\begin{aligned}
& \chi^{\lambda^{*}}\left(\mathcal{C}_{2}\right) \chi^{\lambda^{*}}\left(\mathcal{C}_{N}\right)=(-1)^{\left(\lambda^{1}-1\right) / 2}\left(\begin{array}{c}
\frac{N-2}{2} \\
\frac{\lambda_{1}-2}{2}
\end{array}\right), \quad \text { if } \lambda_{1} \text { is even; } \\
& \chi^{\lambda^{*}}\left(\mathcal{C}_{2}\right) \chi^{\lambda^{*}}\left(\mathcal{C}_{N}\right)=(-1)^{\left(\lambda^{1}+2\right) / 2}\left(\begin{array}{c}
\frac{N-2}{2} \\
\frac{\lambda_{1}-1}{2}
\end{array}\right), \quad \text { if } \lambda_{1} \text { is odd }
\end{aligned}
$$

As for $f^{\lambda^{*}}$, applying the hook length formula we obtain

$$
f^{\lambda^{*}}=\frac{N !}{N \prod_{r=1}^{\lambda_{1}-1} r \prod_{s=1}^{\lambda^{1}-1} s}=\left(\begin{array}{c}
N-1 \\
\lambda_{1}-1
\end{array}\right)
$$

Introducing

$$
\begin{aligned}
& F\left(N, \lambda_{1}\right):=(-1)^{\left(\lambda^{1}-1\right) / 2}\left(\begin{array}{c}
\frac{N-2}{2} \\
\frac{\lambda_{1}-2}{2}
\end{array}\right) /\left(\begin{array}{l}
N-1 \\
\lambda_{1}-1
\end{array}\right) \quad \text { if } \lambda_{1} \text { is even; } \\
& F\left(N, \lambda_{1}\right):=(-1)^{\left(\lambda^{1}+2\right) / 2}\left(\begin{array}{c}
\frac{N-2}{2} \\
\frac{\lambda_{1}-1}{2}
\end{array}\right) /\left(\begin{array}{l}
N-1 \\
\lambda_{1}-1
\end{array}\right) \text { if } \lambda_{1} \text { is odd, }
\end{aligned}
$$

we transform (2.1) into

$$
P_{\gamma}(s)=\frac{1}{N !} \sum_{\lambda_{1}=1}^{N} F\left(N, \lambda_{1}\right) \chi^{\lambda^{*}}(s)
$$


here $\lambda^{*}$ is a hook of size $N$, with the row of length $\lambda_{1}$, and the column of height $\lambda^{1}=$ $N+1-\lambda_{1}$.

Turn to $\chi^{\lambda^{*}}(s)$. Let $s \in \mathcal{C}_{\vec{\nu}}$. Introduce $\ell=\ell(\vec{\nu}):=\min J=\min \left\{j: \nu_{j}>0\right\}$, the size of the smallest positive component of $\alpha$.

Case $\lambda_{1} \leqslant \ell$. Here, for every $\boldsymbol{\alpha}$, there is only one rim hook tableau $T$, since the arm of the hook deleted last must be the whole row of $\lambda^{*}$, and, denoting $\nu=\sum_{j} \nu_{j}$,

$$
\operatorname{ht}(T)=\lambda^{1}-\nu \equiv\left(\lambda^{1}+\nu\right)(\bmod 2) \Longrightarrow \chi^{\lambda^{*}}(s)=(-1)^{\lambda^{1}+\nu} .
$$

Case $\lambda_{1}>\ell$. Since in (2.5) the composition $\boldsymbol{\alpha}$ can be chosen arbitrarily, let us assume that components of $\boldsymbol{\alpha}$ are non-increasing. So the composition $\boldsymbol{\alpha}$ consists of the segment formed by all components of the largest size, followed by the segment formed by all components of the second largest size, and so on, all the way to the terminal segment formed by all components of the smallest size $\ell$. Therefore in a generic tableau $T$ the last rim hook is a diagram $\mu^{*}$ comprising one row and one column of sizes $\mu_{1}$ and $\mu^{1}$, $\mu_{1}+\mu^{1}=\ell+1$. All the other rim hooks in $T$ are either horizontal or vertical, successively deleted from the row and from the column of $\lambda^{*}$ respectively. Let $h_{r}$ be the number of those horizontal hooks of size $r \in J$; so $h_{r} \in\left[0, \nu_{r}\right]$ for $r>\ell$, and $h_{\ell} \in\left[0, \nu_{\ell}-1\right]$. The admissible $\mathbf{h}=\left\{h_{r}\right\}$ must meet the additional constraint

$$
\sum_{r \in J} h_{r} r+\mu_{1}=\lambda_{1}
$$

The total number of the tableaux $T$, with parameters $\mu_{1}, \mu^{1}, \mathbf{h}$, is

$$
\left(\begin{array}{c}
\nu_{\ell}-1 \\
h_{\ell}
\end{array}\right) \prod_{r \in J \backslash\{\ell\}}\left(\begin{array}{c}
\nu_{r} \\
h_{r}
\end{array}\right)=\prod_{r \in J}\left(\begin{array}{c}
\nu_{r}-\delta_{\ell, r} \\
h_{r}
\end{array}\right), \quad \delta_{\ell, r}:=1_{\{r=\ell\}} .
$$

Further, the $\mu^{1}$-long leg of the last hook contributes $\mu^{1}-1$ to the height $\operatorname{ht}(T)$, while the total contribution to $\mathrm{ht}(T)$ of those vertical rim hooks is $\left(\lambda^{1}-\mu^{1}\right)$, the sum of their sizes, minus $\sum_{r}\left(\nu_{r}-\delta_{\ell, r}-h_{r}\right)$, their total number. So

$$
\begin{aligned}
\operatorname{ht}(T) & =\left(\mu^{1}-1\right)+\left(\lambda^{1}-\mu^{1}\right)-\sum_{r \in J}\left(\nu_{r}-\delta_{\ell, r}-h_{r}\right) \\
& \equiv \lambda^{1}-1+\sum_{r \in J}\left(\nu_{r}-\delta_{\ell, r}-h_{r}\right)(\bmod 2) .
\end{aligned}
$$

Therefore the total contribution to $\chi^{\lambda^{*}}(s)$ of the rim hook tableaux $T$ with the last rim hook $\mu^{*}$ is

$$
\begin{aligned}
& (-1)^{\lambda^{1}-1} \sum_{\mathbf{h} \text { meets }(2.14)} \prod_{r \in J}(-1)^{\nu_{r}-\delta_{\ell, r}-h_{r}}\left(\begin{array}{c}
\nu_{r}-\delta_{\ell, r} \\
h_{r}
\end{array}\right) \\
& =(-1)^{\lambda^{1}-1}\left[\xi^{\lambda_{1}-\mu_{1}}\right] \prod_{r \in J} \sum_{h_{r}}(-1)^{\nu_{r}-\delta_{\ell, r}-h_{r}}\left(\xi^{r}\right)^{h_{r}}\left(\begin{array}{c}
\nu_{\ell}-\delta_{\ell, r} \\
h_{r}
\end{array}\right) \\
& =(-1)^{\lambda^{1}-1}\left[\xi^{\lambda_{1}-\mu_{1}}\right] \prod_{r \in J}\left(\xi^{r}-1\right)^{\nu_{r}-\delta_{\ell, r}} .
\end{aligned}
$$


To get $\chi^{\lambda^{*}}(s)$ we need to sum this expression for all $1 \leqslant \mu_{1} \leqslant \min \left\{\ell, \lambda_{1}\right\}$, i.e. for $1 \leqslant \mu_{1} \leqslant \ell$, because $\lambda_{1}>\ell$. Since $\lambda_{1}-\mu_{1} \in\left[\lambda_{1}-\ell, \lambda_{1}-1\right]$,

$$
\begin{aligned}
\chi^{\lambda^{*}}(s) & =(-1)^{\lambda^{1}-1} \sum_{t=\lambda_{1}-\ell}^{\lambda_{1}-1}\left[\xi^{t}\right] \prod_{r \in J}\left(\xi^{r}-1\right)^{\nu_{r}-\delta_{\ell, r}} \\
& =(-1)^{\lambda^{1}-1}\left[\xi^{\lambda_{1}-1}\right]\left(\sum_{\tau=0}^{\ell-1} \xi^{\tau}\right) \prod_{r \in J}\left(\xi^{r}-1\right)^{\nu_{r}-\delta_{\ell, r}} \\
& =(-1)^{\lambda^{1}-1}\left[\xi^{\lambda_{1}-1}\right] \frac{\xi^{\ell}-1}{\xi-1} \prod_{r \in J}\left(\xi^{r}-1\right)^{\nu_{r}-\delta_{\ell, r}} \\
& =(-1)^{\lambda^{1}-1}\left[\xi^{\lambda_{1}-1}\right](\xi-1)^{-1} \prod_{r \in J}\left(\xi^{r}-1\right)^{\nu_{r}} \\
& =(-1)^{\lambda^{1}+\nu}\left[\xi^{\lambda_{1}}\right] \frac{\xi}{1-\xi} \prod_{r \in J}\left(1-\xi^{r}\right)^{\nu_{r}} .
\end{aligned}
$$

Observe that, for $\lambda_{1} \leqslant \ell$, we have $r>\lambda_{1}-1$ for all $r \in J$; so the bottom RHS in (2.15) is

$$
\begin{aligned}
(-1)^{\lambda^{1}+\nu}\left[\xi^{\lambda_{1}-1}\right](1-\xi)^{-1} & \prod_{r \in J}\left(1-\xi^{r}\right)^{\nu_{r}} \\
= & (-1)^{\lambda^{1}+\nu}\left[\xi^{\lambda_{1}-1}\right](1-\xi)^{-1}=(-1)^{\lambda^{1}+\nu},
\end{aligned}
$$

which is the value of $\chi^{\lambda^{*}}(s)$ for $\lambda_{1}<\ell$, see (2.13). Therefore, the bottom line expression in $(2.15)$ for $\chi^{\lambda^{*}}(s)$ holds for all $\lambda_{1}$.

The seemingly unwieldy formula (2.12) for $\mathrm{P}_{\gamma}(s)$ together with (2.15) instantly lead to a promising expression for the marginal distribution of $V_{n}$, the number of cycles of $\gamma$.

Theorem 1. For $N=2 n, \nu \in[1, N]$,

$$
P\left(V_{n}=\nu\right)=\left[x^{N} y^{\nu}\right] \sum_{\lambda_{1}=1}^{N}(-1)^{\lambda^{1}} F\left(N, \lambda_{1}\right)\left[\xi^{\lambda_{1}}\right]\left[\frac{\xi}{1-\xi}\left(\frac{1-x}{1-x \xi}\right)^{y}\right],
$$

or equivalently

$$
E\left[y^{V_{n}}\right]=\sum_{\lambda_{1}=1}^{N}(-1)^{\lambda^{1}} F\left(N, \lambda_{1}\right)\left[\xi^{\lambda_{1}} x^{N}\right]\left[\frac{\xi}{1-\xi}\left(\frac{1-x}{1-x \xi}\right)^{y}\right] .
$$

Proof. By (2.12),

$$
\mathrm{P}\left(V_{n}=\nu\right)=\frac{1}{N !} \sum_{\lambda_{1}} F\left(N, \lambda_{1}\right) \sum_{s: \nu(s)=\nu} \chi^{\lambda^{*}}(s),
$$

and by (2.15) and Cauchy formula

$$
\left|\mathcal{C}_{\vec{\nu}}\right|=N ! \prod_{r} \frac{1}{r^{\nu_{r}} \nu_{r} !}
$$


we have

$$
\begin{aligned}
& \sum_{s: \boldsymbol{\nu}(s)=\vec{\nu}} \chi^{\lambda^{*}}(s)=(-1)^{\lambda^{1}} N !\left[\xi^{\lambda_{1}}\right] \frac{\xi}{1-\xi} \sum_{\substack{\sum_{r} \nu_{r}=\nu, r \nu_{r}=N}} \prod_{r \geqslant 1}\left(\frac{-\left(1-\xi^{r}\right)}{r}\right)^{\nu_{r}} / \nu_{r} ! \\
= & (-1)^{\lambda^{1}} N !\left[\xi^{\lambda_{1}}\right] \frac{\xi}{1-\xi}\left[x^{N} y^{\nu}\right] \prod_{r \geqslant 1}\left[\sum_{\nu_{r} \geqslant 0}\left(\frac{-y x^{r}\left(1-\xi^{r}\right)}{r}\right)^{\nu_{r}} / \nu_{r} !\right] \\
= & (-1)^{\lambda^{1}} N !\left[\xi^{\lambda_{1}}\right] \frac{\xi}{1-\xi}\left[x^{N} y^{\nu}\right] \exp \left(-\sum_{r \geqslant 1} \frac{y x^{r}\left(1-\xi^{r}\right)}{r}\right) \\
= & (-1)^{\lambda^{1}} N !\left[\xi^{\lambda_{1}}\right] \frac{\xi}{1-\xi}\left[x^{N} y^{\nu}\right] \exp \left(-y \log \frac{1}{1-x}+y \log \frac{1}{1-x \xi}\right) \\
= & (-1)^{\lambda^{1}} N !\left[\xi^{\lambda_{1}}\right] \frac{\xi}{1-\xi}\left[x^{N} y^{\nu}\right]\left(\frac{1-x}{1-x \xi}\right)^{y} .
\end{aligned}
$$

The proof is complete.

Note. The reader may wish to compare (2.17) with a formula obtained by Stanley [17] for the univariate generating function of the number of cycles in the product of a fixed cycle $1 \rightarrow 2 \rightarrow \cdots \rightarrow n \rightarrow 1$ and a permutation ranging over a general conjugacy class of $S_{n}$.

Let us use (2.17) to get an explicit formula for $\mathrm{P}\left(V_{n}=\nu\right)$. To begin, from (2.11) it follows that, both for $\lambda_{1}=2 m,(0<m \leqslant n)$ and $\lambda_{1}=2 m+1,(0 \leqslant m<n-1)$,

$$
\begin{aligned}
(-1)^{\lambda^{1}} F\left(N, \lambda_{1}\right) & =(-1)^{n-m+1} \frac{\left(\begin{array}{c}
n-1 \\
m-1
\end{array}\right)}{\left(\begin{array}{c}
2 n-1 \\
2 m-1
\end{array}\right)}=(-1)^{n-m+1} Q(n, m), \\
Q(n, m) & :=\frac{(2 m-1) ! !(2(n-m)-1) ! !}{(2 n-1) ! !}
\end{aligned}
$$

with $(-1) ! !:=1$. Next we evaluate $A\left(n, \nu, \lambda_{1}\right)$, the coefficient of $x^{N} y^{\nu} \xi^{\lambda_{1}}$ in the expansion of $\frac{\xi}{1-\xi}\left(\frac{1-x}{1-x \xi}\right)^{y}$. It is well known, Comtet [3] (Section 5.5), that

$$
\frac{1}{\nu !}\left(\log \frac{1}{1-\eta}\right)^{\nu}=\sum_{\ell \geqslant \nu} \eta^{\ell} \frac{s(\ell, \nu)}{\ell !}
$$

where $s(\ell, \nu)$ is the (un-signed) Stirling number of permutations of $[\ell]$ with $\nu$ cycles. So, setting

$$
\frac{1-x}{1-x \xi}=\frac{1}{1-\eta} \Longleftrightarrow \eta=\frac{x(\xi-1)}{1-x},
$$

we have

$$
\left[y^{\nu}\right]\left(\frac{1-x}{1-x \xi}\right)^{y}=\frac{1}{\nu !}\left(\log \frac{1}{1-\eta}\right)^{\nu}=\sum_{\ell \geqslant \nu} \frac{s(\ell, \nu)}{\ell !}\left(\frac{x(\xi-1)}{1-x}\right)^{\ell} .
$$


Next, for $\ell \leqslant 2 n$,

$$
\left[x^{2 n}\right]\left(\frac{x(\xi-1)}{1-x}\right)^{\ell}=\left[x^{2 n-\ell}\right]\left(\frac{\xi-1}{1-x}\right)^{\ell}=(\xi-1)^{\ell}\left(\begin{array}{c}
-\ell \\
2 n-\ell
\end{array}\right)(-1)^{2 n-\ell},
$$

and finally

$$
\left[\xi^{\lambda_{1}}\right] \frac{\xi}{1-\xi}(\xi-1)^{\ell}=-\left[\xi^{\lambda_{1}-1}\right](\xi-1)^{\ell-1}=(-1)^{\ell-\lambda_{1}+1}\left(\begin{array}{c}
\ell-1 \\
\lambda_{1}-1
\end{array}\right) .
$$

Collecting the pieces we arrive at

$$
\begin{aligned}
A\left(n, \nu, \lambda_{1}\right) & =(-1)^{\lambda_{1}-1} \sum_{\ell \geqslant \nu} \frac{s(\ell, \nu)}{\ell !}\left(\begin{array}{c}
-\ell \\
2 n-\ell
\end{array}\right)\left(\begin{array}{c}
\ell-1 \\
\lambda_{1}-1
\end{array}\right) \\
& =(-1)^{\lambda_{1}-1}\left(\begin{array}{c}
2 n-1 \\
2 n-\lambda_{1}
\end{array}\right) \sum_{\ell \geqslant \nu}(-1)^{\ell} \frac{s(\ell, \nu)}{\ell !}\left(\begin{array}{c}
2 n-\lambda_{1} \\
2 n-\ell
\end{array}\right) .
\end{aligned}
$$

Let $P_{e}\left(V_{n}=\nu\right)$ and $P_{o}\left(V_{n}=\nu\right)$ denote, respectively, the contribution of even $\lambda_{1}$ and odd $\lambda_{1}$ to the RHS of (2.16). To compute $P_{e}\left(V_{n}=\nu\right)$ and $P_{o}\left(V_{n}=\nu\right)$ we will need two simple identities

$$
\begin{aligned}
\sum_{j=0}^{a}(-1)^{j}\left(\begin{array}{l}
a \\
j
\end{array}\right)\left(\begin{array}{c}
2 j \\
b
\end{array}\right) & =(-1)^{a}\left(\begin{array}{c}
a \\
b-a
\end{array}\right) 2^{2 a-b}, \\
\sum_{j=0}^{a}(-1)^{j}\left(\begin{array}{c}
a \\
j
\end{array}\right)\left(\begin{array}{c}
2 j+1 \\
b
\end{array}\right) & =(-1)^{a}\left[\left(\begin{array}{c}
a \\
b-a
\end{array}\right) 2^{2 a-b}+\left(\begin{array}{c}
a \\
b-a-1
\end{array}\right) 2^{2 a+1-b}\right],
\end{aligned}
$$

directly implied by the respective identities

$$
\begin{aligned}
\sum_{b \geqslant 0} x^{b} \sum_{j=0}^{a}(-1)^{j}\left(\begin{array}{c}
a \\
j
\end{array}\right)\left(\begin{array}{c}
2 j \\
b
\end{array}\right) & =\left[1-(1+x)^{2}\right]^{a}=(-1)^{a} x^{a}(2+x)^{a}, \\
\sum_{b \geqslant 0} x^{b} \sum_{j=0}^{a}(-1)^{j}\left(\begin{array}{c}
a \\
j
\end{array}\right)\left(\begin{array}{c}
2 j+1 \\
b
\end{array}\right) & =(-1)^{a}\left(x^{a}+x^{a+1}\right)(2+x)^{a} .
\end{aligned}
$$

(see and compare Gould [8] or www.math.wvu.edu/gould/, Vol.4.PDF, (1.62)). By (2.20), and (2.18),

$$
\begin{aligned}
P_{e}\left(V_{n}=\nu\right)= & \sum_{m=1}^{n}(-1)^{n-m} Q(n, m)\left(\begin{array}{c}
2 n-1 \\
2(n-m)
\end{array}\right) \\
& \times \sum_{\ell \geqslant \nu}(-1)^{\ell} \frac{s(\ell, \nu)}{\ell !}\left(\begin{array}{c}
2(n-m) \\
2 n-\ell
\end{array}\right) \\
= & \sum_{\ell \geqslant \nu}(-1)^{\ell} \frac{s(\ell, \nu)}{\ell !} \sum_{j=0}^{n}(-1)^{j}\left(\begin{array}{c}
n-1 \\
j
\end{array}\right)\left(\begin{array}{c}
2 j \\
2 n-\ell
\end{array}\right) \\
= & \sum_{\ell \geqslant \nu}(-1)^{\ell+n-1} 2^{\ell-2} \frac{s(\ell, \nu)}{\ell !}\left(\begin{array}{c}
n-1 \\
\ell-2
\end{array}\right) .
\end{aligned}
$$


Similarly

$$
\begin{aligned}
P_{o}\left(V_{n}=\nu\right) & =\sum_{\ell \geqslant \nu}(-1)^{\ell} \frac{s(\ell, \nu)}{\ell !} \sum_{j=0}^{n-1}(-1)^{j}\left(\begin{array}{c}
n-1 \\
j
\end{array}\right)\left(\begin{array}{c}
2 j+1 \\
2 n-\ell
\end{array}\right) \\
& =\sum_{\ell \geqslant \nu}(-1)^{\ell+n-1} \frac{s(\ell, \nu)}{\ell !}\left[2^{\ell-2}\left(\begin{array}{c}
n-1 \\
\ell-2
\end{array}\right)+2^{\ell-1}\left(\begin{array}{c}
n-1 \\
\ell-1
\end{array}\right)\right] .
\end{aligned}
$$

Combining (2.21) and (2.22) we have

$$
\begin{aligned}
\mathrm{P}\left(V_{n}=\nu\right) & =\sum_{\ell \geqslant \nu}(-1)^{\ell+n-1} 2^{\ell-1} \frac{s(\ell, \nu)}{\ell !}\left[\left(\begin{array}{c}
n-1 \\
\ell-2
\end{array}\right)+\left(\begin{array}{c}
n-1 \\
\ell-1
\end{array}\right)\right] \\
& =\sum_{\ell \geqslant \nu}(-1)^{\ell+n-1} 2^{\ell-1} \frac{s(\ell, \nu)}{\ell !}\left(\begin{array}{c}
n \\
\ell-1
\end{array}\right) .
\end{aligned}
$$

Therefore

$$
\begin{aligned}
\mathrm{P}\left(V_{n}=\nu\right) & =\frac{1}{2} \sum_{\ell \geqslant \nu} 2^{\ell} \frac{s(\ell, \nu)}{\ell !}\left[x^{n+1-\ell}\right](1+x)^{-\ell} \\
& =\frac{1}{2}\left[x^{n+1}\right] \sum_{\ell \geqslant \nu} \frac{s(\ell, \nu)}{\ell !}\left(\frac{2 x}{1+x}\right)^{\ell}=\frac{1}{2}\left[x^{n+1}\right] \frac{1}{\nu !}\left(\log \frac{1+x}{1-x}\right)^{\nu},
\end{aligned}
$$

by (2.19), as $\frac{2 x}{1+x}=1-\frac{1-x}{1+x}$. Consequently

$$
\mathrm{E}\left[y^{V_{n}}\right]=\frac{1}{2}\left[x^{n+1}\right] \sum_{\nu \geqslant 1} \frac{1}{\nu !}\left(\log \frac{1+x}{1-x}\right)^{\nu}=\frac{1}{2}\left[x^{n+1}\right]\left[\left(\frac{1+x}{1-x}\right)^{y}-1\right],
$$

whence, setting $V_{0}=1$, we get the Harer-Zagier formula (1.2):

$$
\sum_{n \geqslant 0} x^{n+1} \mathrm{E}\left[y^{V_{n}}\right]=\frac{1}{2}\left[\left(\frac{1+x}{1-x}\right)^{y}-1\right] \leftrightarrow 1+2 \sum_{n \geqslant 0} x^{n+1} \mathrm{E}\left[y^{V_{n}}\right]=\left(\frac{1+x}{1-x}\right)^{y} .
$$

\section{Acknowledgments}

I owe a debt of gratitude to Huseyin Acan, Sergei Chmutov and Sergei Duzhin for many discussions of the chord diagrams and of the Harer-Zagier formula. I thank Huseyin, Sergei and Elena Yudovina for very useful critical comments. A referee report definitely helped to improve presentation of the proofs.

\section{References}

[1] S. Chmutov and B. Pittel, The genus of a random chord diagram is asymptotically normal, J. Combin. Theory Ser. A, 120 (2013) 102-110. 
[2] S. Chmutov and B. Pittel, On a surface formed by randomly gluing together polygonal discs, Adv. Appl. Math., 73 (2016) 23-42.

[3] L. Comtet, Advanced Combinatorics, (1974).

[4] P. Diaconis and M. Shahshahani, Generating a random permutation with random transpositions, Z. Wahr. Verw. Gebiete, 57 (1981) 159-179.

[5] P. Diaconis, Group Representations in Probability and Statistics, (1988).

[6] K. Fleming and N. Pippenger, Large deviations and moments for the Euler characteristic of a random surface, Random Struct. Algorithms, 37 (2010) 465-476.

[7] A. Gamburd, Poisson-Dirichlet distribution for random Belyi surfaces, Ann. Probab., 34 (2006) 1827-1848.

[8] H. W. Gould, Combinatorial identities, 2nd Ed., (1972).

[9] J. Harer and D. Zagier, The Euler characteristic of the moduli space of curves, Invent. Math., 85 (1986) 457-485.

[10] C. Itzykson and J. B. Zuber, Matrix integration and combinatorics of modular groups, Comm. Math. Phys. 134 (1990) 197-207.

[11] D. M. Jackson, Counting cycles in permutations by group characters, with an application to a topological problem, Trans. AMS, 299 (1987) 785-801.

[12] S. K. Lando and A. K. Zvonkin, Graphs on Surfaces and Their Applications, (2004).

[13] B. Lass, Démonstration combinatoire de la formule de Harer-Zagier, C. R. Acad. Sci. Paris, 333 (2001) 155-160.

[14] N. Linial and T. Nowik, The expected genus of a random chord diagram, Discrete Comput. Geom., 45 (2011) 161-180.

[15] N. Pippenger and K. Schleich, Topological characteristics of random triangulated surfaces, Random Struct. Algorithms, 28 (2006) 247-288.

[16] R. P. Stanley, Enumerative Combinatorics, Vol. 2, (1999).

[17] R. P. Stanley, Two enumerative results on cycles of permutations, European J. Combinatorics, 32 (2011) 937-943.

[18] D. Zagier, On the distribution of the number of cycles of elements in symmetric groups, Nieuw Arch. Wiskd., 13 (1995) 489-495. 